\title{
Fuzzy Cost based Power Aware QoS Routing Protocol with Mobility Prediction in Mobile Ad Hoc Networks
}

\author{
M.Marimuthu \\ Department of CTA \\ Coimbatore Institute of Technology \\ Coimbatore, India
}

\author{
A.Kannammal \\ Department of Computer Applications \\ Coimbatore Institute of Technology \\ Coimbatore, India
}

\begin{abstract}
Fuzzy Cost Based Power Aware QoS Routing (FCPAQR) protocol proposed to select an optimal path by considering multiple independent QoS metrics. Fuzzy inference rule which is used to calculate the QoS based fuzzy cost of each link to forward the data packets effectively and efficiently. Fuzzy cost is calculated based on various QoS Constraints. QoS constraints can be classified as Time Constraint (Delay and Jitter), Space Constraint (System Buffer), Frequency Constraint (System Bandwidth) Reliability Constraint (Error rate) [9]. Link Expiry Time(LET) and Energy Level are also additionally taken into account. Considering multiple QoS Constraints provide better result than taking single constraint into account.
\end{abstract}

\section{General Terms}

MANETs, Power Aware Protocol, LET

\section{Keywords}

Routing, Fuzzy logic, QoS metrics,

\section{Introduction}

\subsection{MANETs}

Mobile Ad Hoc Network is a collection of wireless nodes and communication is based on the peer-peer fashion. Due to free administration mobile nodes may join or leave at anytime in the network and nodes move frequently across the network. Due to this topology is unpredictable and routing is a challenging one. Laptops, Tablets and Handheld devices are resource limited by its nature. To utilize these types of network resources in proper manner and in order to increase the packet delivery ratio QoS is important for consideration.

The routing protocols proposed for MANETs can be classified as Proactive, Reactive and Hybrid routing. Proactive routing spends more energy on route maintenance [1,2]. Reactive spends more power on route discovery [3,4]. Hybrid routing incorporates the features of the Proactive and Reactive routing $[5,6]$. Lot of protocols is incorporated with QoS metrics. But few researchers considered single QoS Constraint and rest of the researchers has taken double and triples QoS constraint into account

\subsection{Fuzzy Based QoS Routing.}

QoS is usually defined as a set of service requirements that needs to be met by the network while transporting a packet stream from a source to its destination [8]. Costs are the input parameters, which are taken into account and fuzzy logic is applied to calculate the cost. Generally fuzzy inference rule take multiple value as input and generates single value as output. Time constraint consists of Delay and Jitter. Delay is the amount of time takes to transmit a packet from source to destination. The variation in the delay is sometimes called jitter. Space Constraint deals with System Buffer and accommodates the incoming packets from its neighbours. Finally Frequency Constraint concentrates on System Bandwidth refers to width of the frequency band in which it operates.

\section{Related Work}

In Fuzzy Logic Wireless Multipath Routing FLWMR) [10] single QOS metric i.e. hopcount alone is considered for route selection and packet forwarding. Fuzzy Logic Wireless Load Aware Multipath Routing (FLWLAMR) [10] considers network status as a factor for making routing decisions.

The scheme proposed in [11] makes each node to maintain a table to keep the list of the nodes to which it has a connection and the associated bandwidth and delay to reach that neighbor.

A Fuzzy Stochastic Multipath Routing (FSMR) proposed in [12] considers multiple metrics such as energy consumption, buffer occupancy, hop count, battery power and signal strength.

The authors have proposed a multi-objective pareto-optimal technique using Genetic Algorithm (GA) for group communications [13]. Bandwidth, Delay, Jitter, Packet loss rate and blocking property are the five QoS parameters are considered for their model.

In [14] the authors have revealed the superiority of generic fuzzy routing over normal routing approach. They have considered only energy consumption rate at a node, buffer occupancy rate at a node, link stability between the neighboring node and number of intermediate hops in a route.

An Entropy based model proposed for supporting route stability in mobile ad hoc networks [15]. Again entropy value has combined with QoS class and fuzzy logic has been applied to route the packet.

Fuzzy scheduler [16] calculates the priority index of each packet, by considering all the inputs it decides the priority associated with the packet.

The authors have the scheme that incorporates two fuzzy controllers [17] into each node, fuzzy controller1 has three input metric, no of intermediate nodes, packet queue occupancy and internodes distance. While the fuzzy controller 2 predicts the lifetime of the selected route from the source to the destination.

A fuzzy logic based algorithm for finding a bandwidth-delayconstrained by Dijkstra's shortest path algorithm proposed in 
[18]. Bandwidth and delay alone considered as QoS Constraints.

Improved Rank-based Multipath Routing (ImRMR) [19] the authors have proposed a protocol that takes into account of Bandwidth, computing efficiency, power consumption, traffic load and the no of hops as a parameter.

The authors have considered only Bandwidth and Delay as a dual QoS Constraint [20]. For packet forwarding the packet scheduler is used in the architecture as WRR (Weighted Round Robin). In scheduler queues are served according to a configurable weight that can be changed during network operation.

Bandwidth, delay and jitter have considered as a QoS constraints [21]. A flexible QoS multicast routing algorithm based on Artificial Fish Swarm (AFS) algorithm is presented with introduction of principle of fuzzy mathematics.

The authors have proposed a fuzzy scheduler [22] which calculates the priority index of each packet. They consider all the inputs which decide the priority associated with the packet. The fuzzy scheduler uses three input variables to be fuzzified are the expiry time and data rate of the packet and length of the nodes to which the packet is associated with. The output variables are the priority index.

\section{Description of proposed FCPAQR protocol}

As shown in Figure 1, whenever source nodes wants to send a packet to its destination, it first checks in its routing table if path is found, packet is forwarded to the destination. If route is not found source node floods the route request to its intermediate nodes. Intermediate nodes flood the route request to its neighbour nodes. Finally when it reaches the destination route reply send via the route request path to source node. This protocol considers that Delay, Jitter, Queue Length and hop count should be minimum and Node Energy level and LET should be maximum.

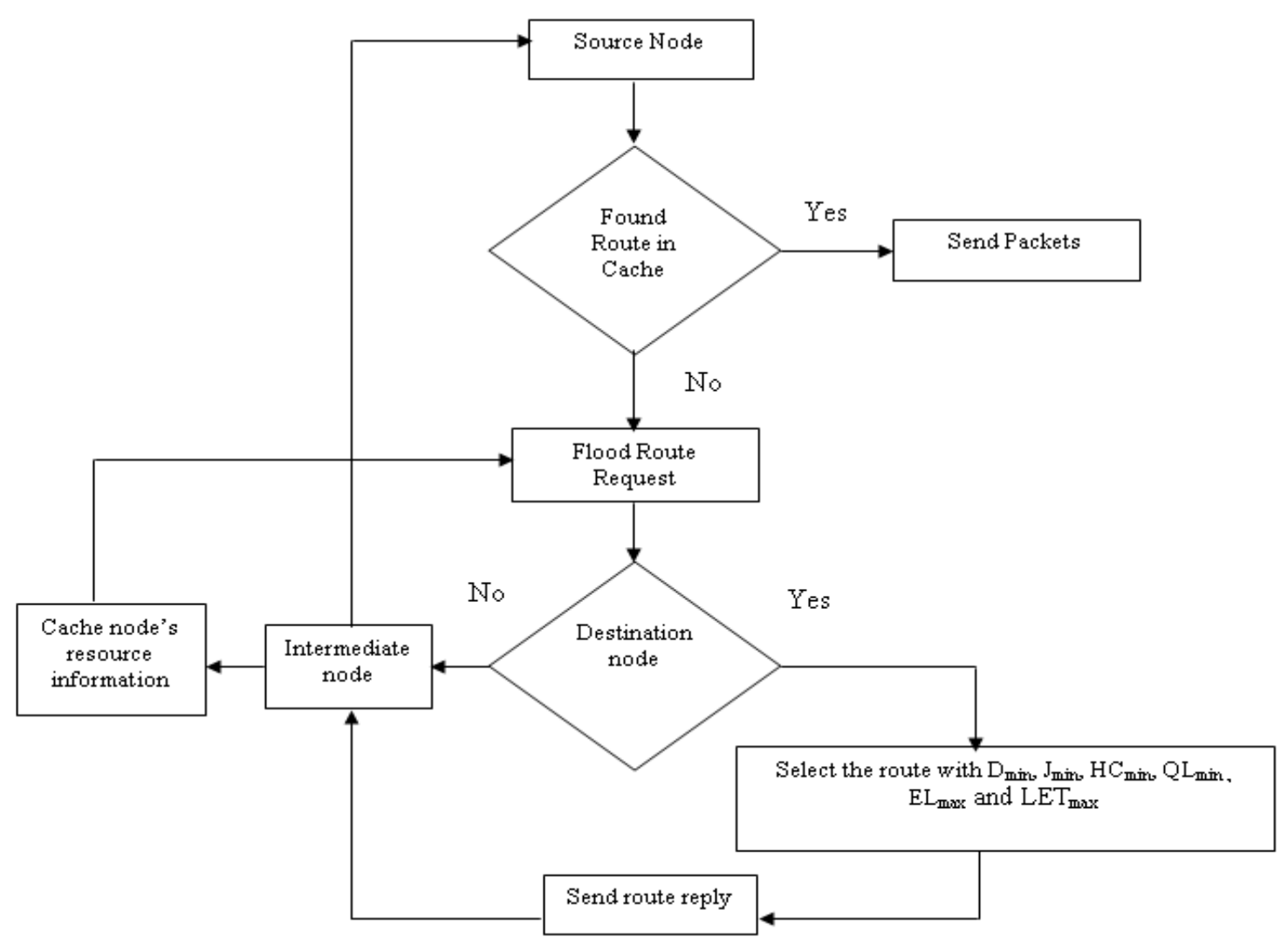

Figure 1 Route finding process in FCPAQR

\section{Illustration}

Figure 2 depicts a graph with QoS metrics for links in FCPAQR protocol. Let Bandwidth $(B)=45$, Delay $(D)=15$, $\operatorname{Jitter}(J)=25, \operatorname{Buffer}(Q)=20, \operatorname{Cost}(C)=40$ and Energy $(E)$ $=75$ respectively. Let the power levels of the nodes $1,2,3,4$, 5,6 and 7 are 95, 90, 90, 93, 95, 85 and 93 respectively. The routes from the source node 1 to destination node 7 are requested. Based on our protocol multiple QoS constraints and for LET the route is calculated. In this example, the path $\mathrm{P}_{1}(1,3,4,6,7)$ do not satisfy the delay and bandwidth constraint. The paths P2 $(1,3,6,7)$ and $\mathrm{P} 3(1,2,4,6,7)$ do not satisfy the delay, jitter and bandwidth constraint. The path P4 $(1,2,4,5,7)$ does not satisfy the delay, jitter and cost constraint. The following paths P5 $(1,2,4,3,6,7)$ and P6 (1, $3,4,2,5,7)$ do not satisfy the delay, jitter, queue and cost constraint. But the paths P7 $(1,2,5,7)$ and P8 $(1,3,4,5,7)$ satisfy delay, jitter, bandwidth, queue and cost constraints. All the above mentioned paths satisfies the power level constraint.

\section{Conclusion}

Mobile Ad Hoc Networks is an active research area for past two decades. Participating nodes are resource limited and energy constrained and packet forwarding requires more 
energy than receiving packets. Energy is a major constraint in these types of networks. Taking energy as a major constraint and designed this protocol with fuzzy logic for effective communication. To show the effectiveness and results of proposed approach, implementation work on Ns2 is still in progress. Future work may incorporate some security mechanism to the protocol.

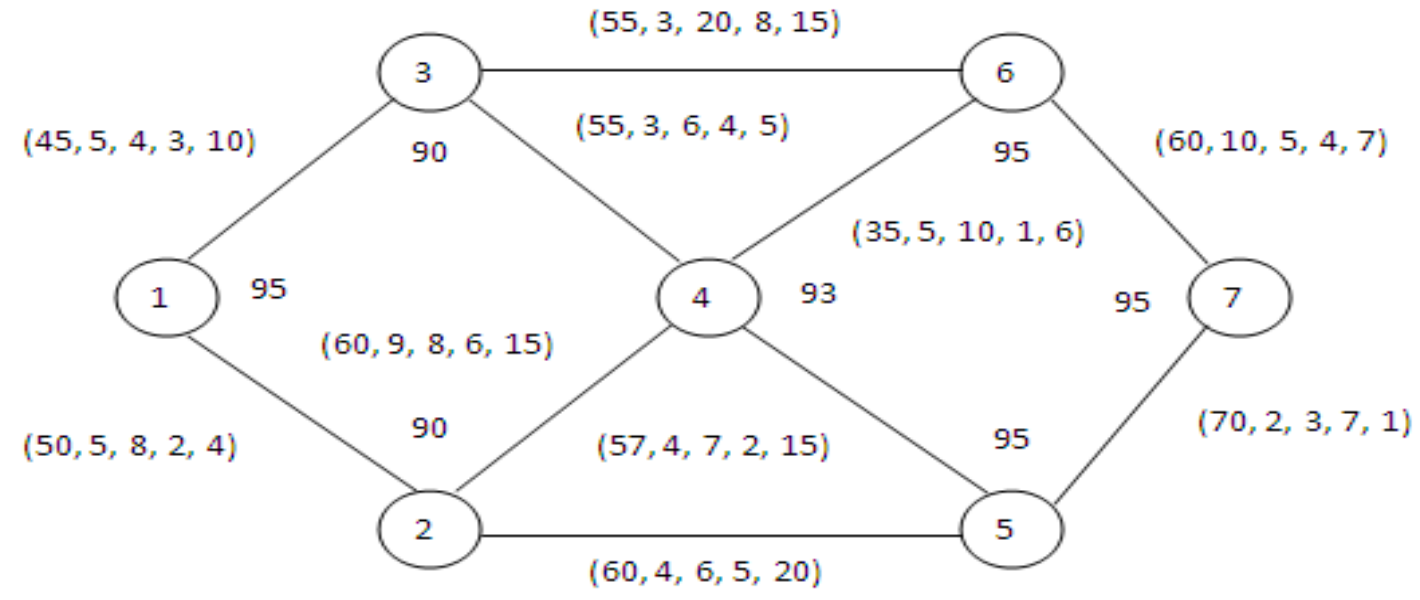

Figure 2 QoS Metrics Graph

\section{References}

[1] Perkins C, Bhagwat P. Highly Dynamic DestinationSequenced Distance-Vector Routing(DSDV) for Mobile Computers. Computer Communications Review 1994; 234-244.

[2] Pei G, Gerla M, Chen T-W. Fisheye State Routing: A Routing Scheme for Ad Hoc Wireless Networks. Proceedings of IEEE Int'l Conf. on Communications (ICC) 2000; 70-74

[3] Johnson D, Maltz D. Dynamic Source Routing in ad hoc wireless networks. Mobile Computing (edited by Imielinski T, Korth H); Kluwer Academic, 1996; 153181

[4] Perkins C, Royer E. Ad-hoc On-Demand Distance Vector Routing. Proceedings of 2nd IEEE Workshop on Mobile Computing Systems and Applications 1999.

[5] Z. Haas and M. Pearlman, "Determining the Optimal Configuration for the Zone Routing Protocol", IEEE JSAC, Special Issue on Ad-Hoc Networks, August 1999.

[6] Venugopalan Ramasubramanian, Zygmunt J. Haas and Emin Gun Sirer, "SHARP: A Hybrid Adaptive Routing Protocol for Mobile Ad Hoc Networks", MobiHoc'03, June 1-3, 2003, Maryland, USA.

[7] E.H.Mamdani and S.Assilian, "An experiment in linguistic synthesis with a fuzzy logic controller", International Journal of Man-Machine Studies, 7(1):113,1975 .

[8] Prasant Mohapatra, Jian Li and Chao Gui, University of California, "QoS in Mobile Ad hoc Networks "IEEE Wireless Communications June 2003 Pages 44 - 52.

[9] Aura Ganz,"Quality Of Service Provision In Mobile Ad Hoc Networks" For TACOM Seminar January 14, 2002 by University Of Massachusetts, Amherst.
[10] Alandjani Gasim, Johnson Eric E. In: Fuzzy routing in ad hoc networks, performance, computing, and communications conference 2003. IEEE international volume, issue, and 9-11 April 2003. p. 525-30.

[11] G. Santhi , Alamelu Nachiappan : Fuzzy-cost based multiconstrained QoS routing with mobility prediction in MANETs, Egyptian Informatics Journal (2012) 13, p.1925

[12] Rajiv V. Dharaskare, M.M.Goswami : Intelligent Multipath Routing Protocol For Mobile Ad Hoc Network, International Journal of computer science and Applications Vol.2, November / December 2009 P. 135 145

[13] Satyananda Champati Rai, Bijan Bihari Misra, Ajit Kumar Nayak, Rajib Mall, Sateesh Kumar Pradhan : A Multi-Objective QoS Optimization with Fuzzy Based Parameter Setting for Real-Time Multicasting, Int. J. Communications, Network and System Sciences, 2010, 3, 530-539.

[14] Hui Liu, Jie Li, Yan-Qing Zhang and Yi Pan : An Adaptive Genetic Fuzzy Multi-path Routing Protocol for Wireless Ad-Hoc Networks, International Conference on Software Engineering, Artificial Intelligence, Networking and Parallel/Distributed Computing and First ACIS International Workshop on Self-Assembling Wireless Networks (SNPD/SAWN'05), 2005, 468 - 475

[15] GUI Chao: An entropy-based stability QoS routing with priority scheduler in MANET using fuzzy controllers, Journal of Communication and Computer, Mar. 2007, Volume 4, No.3 (Serial No.28), 52 - 54.

[16] Shangchao Pi1, Baolin Sun2 : Fuzzy Controllers Based Multipath Routing Algorithm in MANET, on Applied Physics and Industrial Engineering Conference, Physics procedia 24 (2012) $1178-1185$.

[17] Taqwa Odey, Abduladhem A. Ali: Fuzzy Controller Based Stable Routes with Lifetime Prediction in 
MANETs, International Journal of Computer Networks (IJCN), Volume (3) : Issue (1) : 2011, 37 - 42

[18] M. Yaghmaei, M. Baradaran, H. Talebian: A Fuzzy QoS Routing Algorithm for Communication Networks, Communication Systems conference 2006, IEEE, 1 - 5

[19] A Naga Raju, Dr S Rmachandram : Fuzzy Cost Based Multipath Routing For MobileAd-hoc Networks, Journal of Theoretical and Applied Information Technology, 319 -326 .

[20] B. Sun, C. Gui, Q. Zhang, H. Chen: Fuzzy Controller Based QoS Routing Algorithm with a Multiclass Scheme for MANET, Int. J. of Computers, Communications \&
Control, ISSN 1841-9836, E-ISSN 1841-9844 Vol. IV (2009), No. 4, pp. 427-438

[21] Junwei Wang Zhaoxia Wu: A Fuzzy Decision Based Intelligent QoS Multicast Routing Algorithm , Automation and Logistics Conference, Chongqing, China, August 2011, 169 - 172

[22 ]ChunHua Xia: An Entropy-Based Stability QoS Routing with Priority Scheduler in MANET, Wireless Communications Networking and Mobile Computing (WiCOM), Conference on Date: 23-25 Sept. 2010, 1-3 